\title{
Modelación de la producción y el decaimiento del oxígeno disuelto en embalses
}

\author{
J.L. de Victorica-Almeida \\ Coordinación de Ingeniería Ambiental \\ Instituto de Ingeniería, UNAM \\ E-mail:jva@pumas.iingen.unam.mx
}

(recibido: mayo de 2002; aceptado: noviembre de 2002)

\begin{abstract}
Resumen
Este trabajo plantea un modelo explícito en un esquema de diferencias finitas para estudiar la evolución del oxígeno disuelto $(O D)$ en embalses. Está conformado por las ecuaciones de transporte y cinéticas de los principales procesos de producción y decaimiento del $O D$ dentro del agua. Se considera como fuente de $O D$ el que se produce vía fotosíntesis y como sumideros, el $O D$ requerido para la respiración de los microorganismos que llevan a cabo la degradación del material orgánico, tanto del que ingresa por los tributarios, como para el que se genera dentro de él por la muerte del fitoplancton. En las ecuaciones cinéticas se consideran los efectos de la luz y la temperatura. Al modelo, aunque se conformó específicamente para analizar la evolución del $O D$, se le pueden hacer ajustes para incorporarle las ecuaciones que describen la cinética de otros parámetros de la calidad del agua, como son nitrógeno y fósforo, que están íntimamente relacionados con el proceso de eutrofización, fenómeno característico en la mayoría de los embalses mexicanos.
\end{abstract}

Descriptores: embalses, $O D, D B O$, fotosíntesis

\begin{abstract}
In this work, an explicit model in a scheme of finite differences is established to study the evolution of dissolved oxygen $(D O)$ in reservoirs. It is conformed by the kinetic and transport equations of the main processes of DO production and decay within the water. The DO production process is photosynthesis and the process of decay is the DO used by the respiration of organic matter degrading microorganisms, both as inputs and by the death of phytoplancton. The kinetic equations consider the effects of light and temperature. This model, although specifically designed to analyze the evolution of $D O$, can be adjusted to include the equations that describe the kinetics of several water quality parameters, such as nitrogen and phosphorous, which are closely related with the process of eutrophication, a characteristic phenomenon in most Mexican reservoirs.
\end{abstract}

Keywords: Reservoirs, BOD, DO, photosynthesis

\section{Introducción}

El conjunto de características físicas químicas y biológicas de un determinado tipo de agua define su calidad. En los embalses, éstos componentes no permanecen estáticos por considerarse sistemas abiertos, pero tienen una dinámica par- 
de compensación $I_{c}$ igual a la de $I_{o}$ en la que la magnitud de $C_{m}$ iguala a la de la tasa de mortandad $D_{p}$ del fitoplancton, y por ende, la producción neta de oxígeno debida a la fotosíntesis es cero.

De acuerdo con lo anterior, para la ecuación 3 es necesario establecer los siguientes límites:

$$
\begin{array}{lll}
\mathrm{F}(I)_{(\mathrm{z})}=1 & \text { para } & I_{o}>I_{s} \\
\mathrm{~F}(I)_{(\mathrm{z})}=0 & \text { para } & I_{0} \leq I_{c}
\end{array}
$$

Por otra parte, $I_{0}$ depende de la región geográfica y características de la atmósfera donde se ubique el embalse, y su magnitud puede estimarse de diversas formas, dependiendo del grado de precisión deseada e información disponible; así, para algunos propósitos basta con hacer mediciones continuas durante algún período de interés y obtener su promedio, o usar, si existe, la información de radiación solar global que reporte la literatura para la zona en estudio. Otra manera de estimar la variabilidad de $I_{0}$ es mediante algún modelo de radiación, como el estructurado en el Instituto Mexicano de Tecnología del Agua (De Victorica, 1988), el cual con base en algunas de las relaciones obtenidas por Ryan y Harleman (1973) y por la TVA Engineering Laboratory (1972), permiten cuantificar la magnitud de $I_{0}$ con suficiente aproximación, desde el punto de vista práctico, para cualquier localidad geográfica y día del año, requiriendo para ello, información del tipo que normalmente se obtiene en las estaciones climatológicas y meteorológicas. En las referencias arriba señaladas puede observarse con detalle la estructura del modelo, aquí para limitar la extensión del artículo, solamente se presenta su forma y en la lista de símbolos, el significado de los parámetros contenidos en él:

$$
\begin{gathered}
I_{0}=\left(1-0.65 N^{2}\right)\left(1-R_{(t)}\right) a_{0} \\
\frac{a^{\prime \prime}+0.5\left(1-a^{\prime}-d\right)-0.5 d a}{1-0.5 R_{g}\left(1-a^{\prime}+d s\right)}
\end{gathered}
$$

Por otro lado, debido a que casi toda la radiación solar fuera del rango visible 400nm-770nm - (Reid y Wood, 1976) que llega a la superficie del agua, se absorbe en los primeros centímetros bajo la superficie, conviene introducir un factor $\beta$, adimensional, que considere dicha absorción (TVA, 1972):

$$
\beta=\frac{I_{a}}{I_{0}}
$$

donde $I_{a}$, es la radiación solar total que se absorbe, e igual a $\beta \bullet I_{o}$, por lo que la radiación que penetra será $(I-\beta) I_{0}$. Reemplazando en la ecuación 3 el término $I_{0}$ por esta relación, se tendrá una ecuación más precisa para calcular la magnitud de la radiación solar que penetra en el agua.

Por su parte, los parámetros $\beta$ y $\eta$ dependen de las propiedades ópticas del agua, como la turbiedad, contenido de material particulado en suspensión y el color; por ello, sus valores deben ser medidos y no supuestos. En la tabla 1 se presentan algunos valores de $\beta$ y $\eta$ para varios tipos de agua (TVA, 1972).

En lo que concierne a la temperatura, para tomar en cuenta sus efectos sobre la rapidez con que proceden los procesos biológicos, se han formulado diversas ecuaciones; de ellas, la expresión termodinámica de Vant't Hoff-Arrhenius es la 
Tabla 1. Valores de los coeficientes $\beta$ y $\eta$ para algunos tipos de agua

\begin{tabular}{lcc}
\hline \multicolumn{1}{c}{ TIPO DE AGUA } & $\begin{array}{c}\beta \\
\text { (adimensional) }\end{array}$ & $\begin{array}{c}\eta \\
(1 / \mathrm{m})\end{array}$ \\
\hline Agua pura & 0.63 & 0.052 \\
Agua oceánica clara & 0.64 & 0.081 \\
$\begin{array}{l}\text { Agua oceánica } \\
\text { (promedio) }\end{array}$ & 0.68 & 0.122 \\
Agua marina costera & 0.69 & 0.325 \\
(promedio) & 0.69 & 0.425 \\
Agua marina costera & 0.58 & 0.720 \\
turbia & 0.50 & 1.400 \\
Lago Mendota & 0.24 & 1.110 \\
Lago Trout & & \\
Lago Big Ridge & &
\end{tabular}

más aceptada, y establece que el cambio en la rapidez con que se efectua una reacción química o bioquímica, por efecto de la temperatura, puede cuantificarse con la siguiente ecuación (Metcalf y Eddy, 1979):

$$
k_{2}=k_{1} \theta^{\left(T_{2}-T_{1}\right)}
$$

En la ecuación anterior las $k^{\prime}$ s son las tasas de reacción evaluadas a las temperaturas $\mathrm{T}_{1} \mathrm{y}_{\mathrm{T}}$; y $\theta$, habitualmente llamado coeficiente de temperatura, es un parámetro característico para cada población microbiana, por lo que debe medirse o seleccionarse con precaución.

Ahora, definiendo $T_{2}=T_{(z)}$ en el término $\theta^{\left(T_{2}-T_{1}\right)}$ contenido en la ecuación 6 , se obtienen los coeficientes $F(T)_{(z)}$ y $F_{l}(T)_{(z)}$ de las ecuaciones (1) y (2):

$$
\begin{gathered}
F(T)_{(z)}=\theta^{\left[T_{(z)}-25\right]} \\
F_{1}(T)_{(z)}=\theta_{1}^{\left[T_{(z)}-25\right]}
\end{gathered}
$$

para corregir por temperatura las tasas de crecimiento $C_{m}$ y mortandad $D_{p}$ del fitoplancton y de decaimiento $k_{1}$ de la $D B O$, respectivamente.

En las ecuaciones anteriores, $T_{(z)}$ es la temperatura a una determinada profundidad en la columna de agua del embalse; y dado que estos sistemas son abiertos, intercambian masa y energía con su entorno, por lo que su perfil térmico es variable.

Tal variabilidad puede conocerse haciendo mediciones rutinarias de la temperatura en la columna de agua, o mediante algún modelo térmico; por ejemplo, la ecuación de difusión térmica unidimensional, la cual es de utilidad práctica para determinar el comportamiento térmico de embalses en los que no existan descargas de agua caliente y en los que las corrientes convectivas generadas por los afluentes y efluentes de agua sólo sean de importancia en su entorno, y por tanto, puedan ignorarse. La ecuación referida, en diferencias finitas, es (Tabor y Weinberger, 1981):

$$
T_{i, m+1}=\frac{\Delta t}{\rho_{i, m} C_{P i, m} \Delta Z^{2}}\left[\frac{1}{4}\left(K_{i+1, m}-K_{i-1, m}\right)\left(T_{i+1, m} T_{i-1, m}\right)+\right.
$$


$\left.K_{i, m}\left(T_{i+1, m}-2 T_{i, m}+T_{i-1, m}\right)+\left(I_{i-\frac{1}{2}}^{1}-I_{i+\frac{1}{2}}\right) \Delta Z\right]+T_{i, m}$

donde $i \Delta Z=Z ; m \Delta t=t$

Para la solución numérica de la ecuación anterior, las condiciones iniciales quedan definidas al proporcionar la distribución inicial de la temperatura en la columna de agua; es decir, al dar los valores de $T(z, t=0)$ y para las condiciones en las fronteras mediante un balance de calor entre radiación solar neta $I_{0}$ que llega a la superficie del agua, según la ecuación 4, la que sale de ella, $\phi_{b r}$ y los calores $\phi_{e} y \phi_{c}$ que se pierden por evaporación y conducción, haciendo la consideración de que las pérdidas o ganancias de calor en las fronteras rígidas pueden ignorarse. Los flujos de calor $\phi_{b r}, \phi_{\ell}$ y $\phi_{c}$ pueden calcularse con las siguientes relaciones (Hiriart y Pereyra, 1980):

$$
\begin{gathered}
\phi_{b r}=5.49 \times 10^{-8}\left(T_{s}-273.4\right)^{4} \\
\phi_{c}=4.99 w\left(e_{s}-e_{a}\right) \\
\phi_{c}=2.29 w\left(T_{s}-T_{a}\right)
\end{gathered}
$$

En las ecuaciones anteriores $\mathrm{T}_{s} \mathrm{y}_{a}$ son las temperaturas en la superficie del agua y en el aire, respectivamente. Para calcular las presiones de vapor $\mathrm{e}_{a}$ y $\mathrm{e}_{s}$ en el aire y agua, pueden usarse las siguientes expresiones (Hiriart y Pereyra, 1980):

$$
\begin{array}{r}
e_{a}=(H / 100)\left(162.9 \times 10^{6} \exp \right. \\
\left.\left(-4157 /\left(T_{a}+239\right)\right)\right) \\
e_{s}=162.9 \times 10^{6} \exp \left(4157 /\left(T_{s}+239\right)\right)
\end{array}
$$

donde $\mathrm{H}$ es la humedad relativa.

\section{Ecuaciones de continuidad}

Las ecuaciones de continuidad resultan de hacer, en cualquier volumen de control, un balance de masas; así, de acuerdo con el esquema y nomenclatura de la figura 1, las ecuaciones de continuidad, en un esquema de diferencias finitas para analizar la evolución del $O D$ y la $D B O$ en un embalse con una relación pequeña entre el ancho de su superficie libre y su longitud como el que aquí se estudia son:

$$
\begin{gathered}
O D_{(i, I)}^{(t+1)}=O D_{(i, I)}^{(t)} \\
+\left[\bar{B}_{(i, J-1)} U_{(i, I-1)}^{(t)} O D_{(i, I-1)}^{(t)}-\bar{B}_{(i, J)} U_{(i, I)}^{(t)} O D_{(i, I)}^{(t)}\right] \frac{\Delta Z \Delta t}{\forall_{(i, I)}} \\
+\left[\bar{B}_{(i, j-I)} U_{v(i-1, I)}^{(t)} O D_{(i-1, I)}^{(t)}-\bar{B}_{(i-1, j-1)} U_{v(i, I)}^{(t)} O D_{(i, I)}^{(t)}\right] \frac{\Delta L \Delta t}{\forall_{(i, I)}} \\
+\left[\alpha\left(C_{m} F(I)_{(z)}-D_{p}\right) F(T)_{(z)} P_{(i, I)}^{(t)}-k_{1} D B O_{(i, I)}^{(t)} F_{1}(T)_{(z)}\right] \Delta t
\end{gathered}
$$

$$
\begin{gathered}
D B O_{(i, I)}^{(t+1)}=D B O_{(i, I)}^{(t)} \\
+\left[\bar{B}_{(i, J-1)} U_{(i, I-1)}^{(t)} D B O_{(i, I-1)}^{(t)}-\bar{B}_{(i, J)} U_{(i, I)}^{(t)} D B O_{(i, I)}^{(t)}\right] \frac{\Delta Z \Delta t}{\forall_{(i, I)}} \\
+\left[\bar{B}_{(i, j-1)} U_{v(i-1, I)}^{(t)} D B O_{(i-1, I)}^{(t)}-\bar{B}_{(i-1, j-1)} U_{v(i, I)}^{(t)} D B O_{(i, I)}^{(t)}\right] \frac{\Delta L \Delta t}{\forall_{(i, I)}}
\end{gathered}
$$$$
+\left[k_{1} D B O_{(i, I)}^{(t)} F_{1}(T)_{(z)}+\alpha_{1}\left(D_{p} F(T)_{(z)} P_{(i, I)}^{(t)}\right)\right] \Delta t
$$

En las ecuaciones anteriores, es necesario reemplazar los términos que representan los cambios por reacción (último término del lado derecho), por las ecuaciones que producen dichos cambios en cada intervalo de tiempo $\Delta \mathrm{t}$. Así, si se define a los términos de reacción como $A$ y $B$ en las ecuaciones 15 y 16 respectivamente, y si además se considera que se trata de reacciones cinéticas de primer orden, para la ecuación 15 se tiene: 

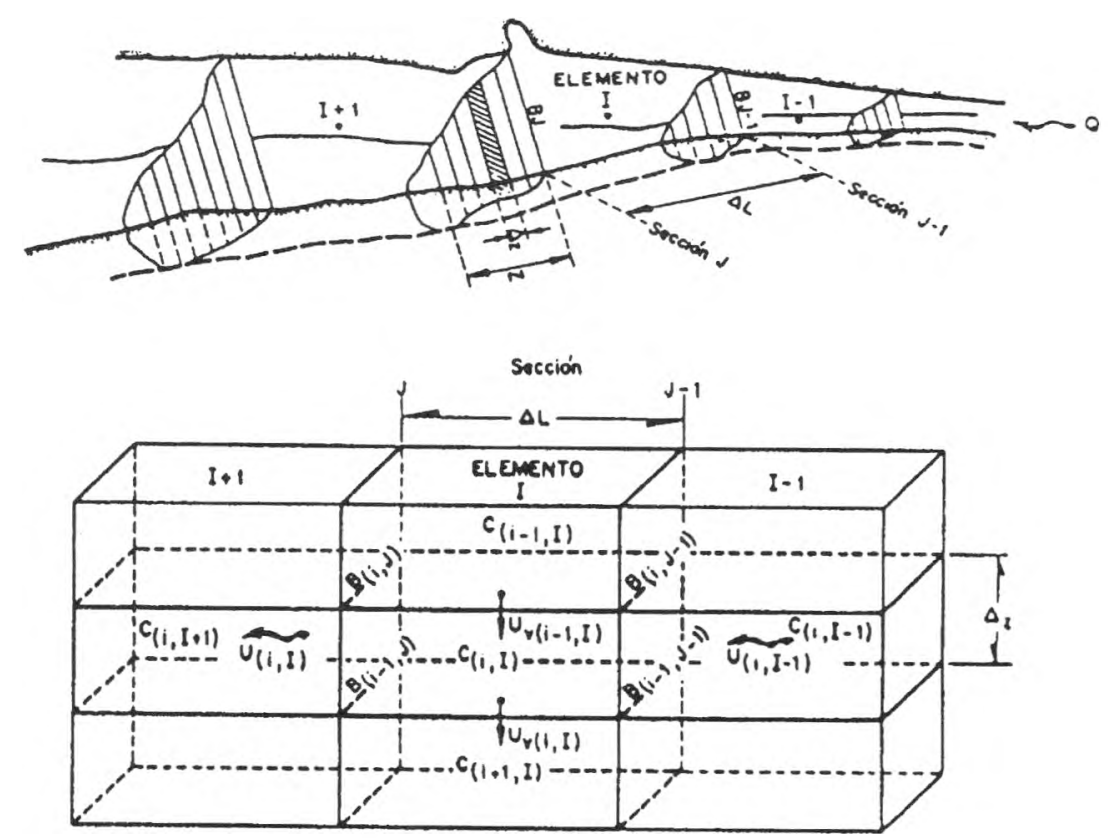

Figura 1. Esquema conceptual utilizado para el planteamiento de las ecuaciones de continuidad

$$
\begin{gathered}
A=\alpha\left\{P_{(i, I)}^{(t)} \exp \left(C_{m} F(I)_{z} F(T)_{(z)} \Delta t\right)\right. \\
\left.-P_{(i, I)}^{(t)}\left\lfloor 1-\exp \left(-D_{p} F(T)_{(z)} \Delta t\right)\right\rfloor\right\} \\
-D B O_{(i, I)}^{(t)}\left[1-\exp \left(-k_{1} F_{1}(T)_{(z)} \Delta t\right)\right]
\end{gathered}
$$

y para la ecuación 16:

$$
\begin{aligned}
& B=-D B O_{(t, l)}^{(t)}\left\lfloor 1-\exp \left(-k_{1} F_{1}(T)_{(z)} \Delta t\right)\right\rfloor \\
& +\alpha_{1}\left\{P_{(i)}^{(t)}\left\lfloor 1-\exp \left(-D_{p} F(T)_{(z)} \Delta t\right)\right\rfloor\right\}
\end{aligned}
$$

Sustituyendo, respectivamente, las ecuaciones 17 y 18 por los términos de reacción en las ecuaciones 15 y 16 , se tendrá un sistema que permita conocer la evolución del oxígeno disuelto y de la demanda química de oxígeno en el embalse.

\section{Hidrodinámica}

De acuerdo con las ecuaciones 15 y 16 , para simular la evolución del oxígeno disuelto y la demanda bioquímica de oxígeno dentro del embalse, además de los parámetros cinéticos involucrados, es necesario conocer el campo de velocidades. Para ellos se propuso como hipótesis que se trata de un flujo cuasipermanente, que el gasto que entra al embalse es igual al que sale durante cada período de simulación y que el nivel del agua dentro del vaso se mantiene constante, o sea, que no hay cambio en el almacenamiento.

También, si el ancho de las secciones transversales del embalse son pequeñas en comparación con su longitud, se acepta un campo de flujo en dos dimensiones, suponiendo una distribución de velocidades sensiblemente vertical y horizontal en todas las secciones del embalse.

Para determinar las velocidades horizontales, se empleó el criterio de Kikkawa (1978) donde se considera que la distribución de velocidades es uniforme y que se cumple el principio de continuidad para flujo permanente en cualquier 
volumen de control. D e acuerdo con lo anterior y el esquema de la figura 2 , la velocidad media con que ingresa el agua al elemento de volumen por la sección $J$ es:

$$
U_{(J)}=\frac{Q}{A_{(J)}}
$$

Donde $Q$ es el flujo volumétrico que pasa por unidad de tiempo a través de la sección $J ; A_{(J)}$ es el área hidráulica total de la sección correspondiente, cuya magnitud esta definida por:

$$
A_{(J)}=0.5\left(B_{(s, j)}+B_{(i, j)}\right) \Delta Y_{(J)}
$$

Donde $B_{(s, j)}$ y $B_{(i, j)}$ son los anchos de la sección en la parte superior e inferior, respectivamente.

De igual manera, la velocidad con que sale el agua por la sección $J+1$ es:

$$
U_{(J+1)}=\frac{Q}{A_{(J+1)}}
$$

En lo que concierne a las velocidades en el sentido vertical, éstas quedan definidas al aplicar la ecuación de continuidad para flujo permanente, en cualquier celda (i) de un elemento (I); así, de acuerdo con nomenclatura de la figura 1 se obtiene:

$$
U_{V_{(i, I)}}=\frac{Q_{(i, I-1)}-Q_{(i, I)}+Q_{V_{(i-1, I)}}}{A_{h i_{(i, I)}}}
$$

En la ecuación anterior, los términos $Q_{(i, I-1)}, Q_{(i, I)}$ son los gastos de ingreso y egreso por las secciones transversales a la celda (i) del elemento I; $Q_{V_{(i-1, l)}}$ representa el gasto que ingresa a la celda (i) por la sección horizontal superior que la confina, y $A_{h i_{(i, I)}}$ es el área de la sección horizontal inferior de la celda (i), definida como:

$$
A_{h i_{i, i, 1}}=0.5\left(B_{(i-1, j-1)}+B_{(i-1, j)}\right) \Delta L
$$

Cabe señalar que el término $Q_{V_{(1-1,1)}}$ que aparece en el numerador de la ecuación 22, queda definido al aplicar la ecuación de continuidad para flujo permanente en la

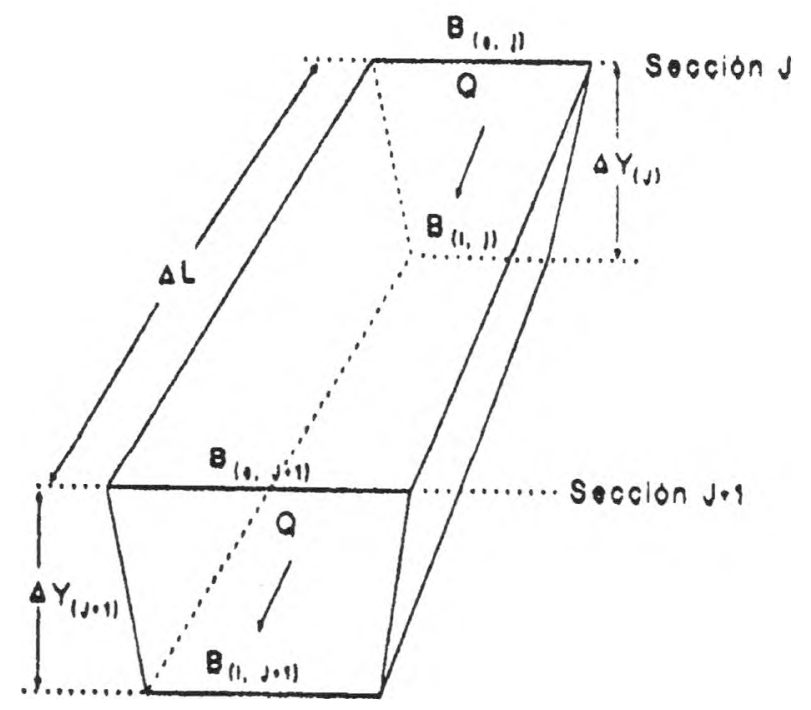

Figura 2. Esquema utilizado para el cálculo de las velocidades 
celda $C_{(i-1, l)}$, cuya sección horizontal superior sea la superficie libre del agua; así, de acuerdo con el esquema y nomenclatura de la figura 1 el término $Q_{V_{(i-1, l)}}$ queda definido como:

$$
Q_{V_{(i-1, l)}}=Q_{(i-1, I-1)}-Q_{(i-1, l)}
$$

Finalmente, la magnitud de los términos del lado derecho de la ecuación anterior, se obtienen mediante el producto de las velocidades calculadas con las ecuaciones 19 y 21, y el área de las secciones transversales que confinan a la celda $C_{(i-1, l)}$.

\section{Secuencia de cálculo}

a) En la etapa inicial $(\mathrm{t}=0)$, además del intervalo de tiempo $\Delta$ t entre cada etapa de cálculo, deben proporcionarse las magnitudes de los parámetros $\alpha, \alpha_{1}, C_{m}, D_{p}$ $k_{1}, I_{s}, I_{c}, \beta, \eta, \theta, T_{a}, T_{s}$ las áreas hidráulicas de las secciones de las celdas y elementos en que se discretice el embalse, los ingresos de agua, las concentraciones de $O D, D B O$ y densidad fitoplanctónica $P$, tanto en el agua influente a la presa como en la columna de agua de la misma. Tambien, deben darse los valores de $I_{0}$ y de $T_{(z)}$, que pueden calcularse con las ecuaciones 4,5 y de la 9 a la 14, o haciendo mediciones rutinarias de tales parámetros.

b) Se calculan, con las ecuaciones 3 , 7 y 8 , las magnitudes de $F(I)_{(z)}, F(T)_{(z)}$ y $F_{l}(T)_{(z)}$.

c) Con la información obtenida en los pasos ayb se resuelven las ecuaciones 17 y 18 .

d) Con las ecuaciones 19 a 24 se obtienen los volúmenes y velocidades en cada una de las celdas que conformen los elementos.

e) Para el caso de un embalse en el que solamente se tenga un afluente y un efluente, con la información de los pasos anteriores, se logran las concentraciones de $O D$ y $D B O$ para el tiempo $\mathrm{t}+\mathrm{l}$ con las ecuaciones 15 y 16.

f) Se actualizan los valores, tanto del o los gastos de ingreso al embalse, sus concentraciones de $O D, D B O$ y $P$, como las magnitudes de $I_{0}$ y $T_{(i)}$, ya que estos pueden cambiar su valor en cada paso de tiempo $\Delta t$ o al término de cierto número de etapas de cálculo, como cuando se usan las magnitudes diarias medias mensuales de tales parámetros.

g) Finalmente, tomando como base para la siguiente etapa de cálculo los resultados obtenidos en el inciso e y tomando en cuenta los del inciso $f$, se retorna al paso $\mathbf{b}$.

\section{Lista de símbolos}

$A_{(, j)} \quad$ área hidráulica de la sección j, $\left[L^{2}\right]$

$\mathrm{A}_{\mathrm{hi}(\mathrm{i}, \mathrm{I})}$ área hidráulica de la sección horizontal de la celda i del elemento I, $\left[L^{2}\right]$

$a^{\prime} \quad$ coeficiente medio de trasmisión atmosférica después de la dispersión, [adim]

$a^{\prime \prime}$ coeficiente medio de trasmisión atmosférica después de la dispersión y absorción, [adim]

$B_{(i, j)}$ ancho inferior de la sección $\mathrm{j},[\mathrm{L}]$

$B_{(s, j)}$ ancho superior de la sección j, [L]

$\mathrm{B}_{m(\mathrm{i}, \mathrm{j})}$ anchomedio dela sección $\mathrm{j}$ dela celda I, [L] 
$C_{m} \quad$ tasa máxima de crecimiento para comunidades heterogéneas de fitoplancton, $\left[T^{-1}\right]$

$\mathrm{C}_{\mathrm{p}} \quad$ capacidad calorífica del agua a presión constante, $\left[E M^{-1} K^{-1}\right]$

$D B O$ demanda bioquímica de oxígeno, $\left[M L^{-3}\right]$

$D_{p} \quad$ tasa máxima de mortandad para comunidades heterogéneas de fitoplancton, $\left[T^{-1}\right]$

d coeficiente de atenuación de los rayos solares debido a la absorción y dispersión causadas por el polvo, [adim]

da coeficiente de atenuación de los rayos solares debido a la absorción causada por el polvo, [adim]

ds coeficiente de atenuación de los rayos solares debido a la dispersión causada por el polvo, [adim]

$e^{a} \quad$ presión de vapor en el aire, $\left[N L^{-2}\right]$

$e^{s} \quad$ presión de vapor en el agua, $\left[N L^{-2}\right]$

$F(I)_{(z)}$ coeficiente para ajustar a $C_{m}$ por efectos de la intensidad de la luz en la columna de agua, [adim]

$F(T)_{(z)}$ coeficiente para ajustar a $C_{m}$ y $D_{p}$ por efectos de la temperatura en la columna de agua, [adim]

$F_{l}(T)_{(z)}$ coeficiente para ajustar a $k_{1}$ por efectos de la temperatura en la columna de agua, [adim]

$\mathrm{H}$ humedad relativa, [adim]

I designación del elemento de discretización del embalse, [adim]

$I_{a} \quad$ radiación solar total que se absorbe en la superficie del agua, $\left[E L^{-2} T^{-1}\right]$

$I_{c} \quad$ intensidad de compensación dela luz para una comunidad heterogénea de fitoplancton, $\left[E L^{-2} T^{-1}\right]$

$I_{0} \quad$ intensidad de la radiación solar neta que llega a la superficie del agua, $\left[E L^{-2} T^{-1}\right]$
Is intensidad de saturación de la luz para una comunidad heterogénea de fitoplancton, $\left[E L^{-2} T^{-1}\right]$

i designación de la celda del elemento I, [adim]

j designación de la sección hidráulica, [adim]

$k^{1} \quad$ tasa de decaimiento de la $D B O$ a la temperatura $\mathrm{T}_{1},\left[\mathrm{~T}^{-1}\right]$

$k^{2} \quad$ tasa de decaimiento de la $D B O$ a la temperatura $\mathrm{T}_{2},\left[\mathrm{~T}^{-1}\right]$

$\mathrm{K}_{i, m} \quad$ conductividad térmica, $\left[E L^{-1} T^{-1} K^{-1}\right]$

$\mathrm{N}$ fracción del cielo cubierto por las nubes, [adim]

OD concentración de oxígeno disuelto, $\left[M L^{-3}\right]$

$P \quad$ concentración de fitoplancton, $\left[L^{-3}\right]$

Q flujo volumétrico, $\left[L^{3} T^{-1}\right]$

$q_{0}$ radiación solar extraterrestre, $\left[E L^{-2} T^{-1}\right]$

$R_{g} \quad$ reflectividad total del suelo en el entorno del embalse, [adim]

$R_{(t)} \quad$ reflectividad total dela superficie del agua, [adim]

$\mathrm{T}_{a} \quad$ temperatura del aire, $[\mathrm{K}]$

$\mathrm{T}_{s} \quad$ temperatura en la superficie del agua, [K]

$\mathrm{T}_{(z)}$ temperatura del agua en la profundidad $z,[\mathrm{~K}]$

t tiempo, [T]

$\mathrm{U}_{(i, \mathrm{I})}$ velocidad del agua en la celda $i$ del elemento I, $\left[L T^{-1}\right]$

$\mathrm{U}_{\mathrm{h}(i, \mathrm{I})}$ velocidad del agua en el sentido horizontal que sale de la celda $i$ del elemento I, $\left[L T^{-1}\right]$

$U_{v(i, I)}$ velocidad del agua en el sentido vertical que sale de la celda $i$ del elemento I, [ $\left.L T^{-1}\right]$

$\forall_{(i, I)} \quad$ volumen de la celda $i$ del elemento I, $\left[L^{3}\right]$

w velocidad del viento, $\left[L T^{-1}\right]$

$z \quad$ profundidad, [L] 
$\alpha \quad$ constante estequiométrica, OD/No. de organismos, $\left[\mathrm{ML}^{-3}\right]$

$\alpha_{1} \quad$ constante estequiométrica, $D B O /$ No. de organismos, $\left[M L^{-3}\right]$

$\beta \quad$ fracción de $I_{0}$ que se absorbe en la superficie del agua, [adim]

$\Delta \mathrm{L}$ distancia entre dos secciones hidráulicas contiguas, [L]

$\Delta \mathrm{t}$ intervalo de tiempo, [T]

$\Delta Y$ distancia entre los anchos que delimitan la sección hidráulica de cualquier elemento I, [L]

$\Delta \mathrm{Z}$ distancia entre los anchos que delimitan la sección hidráulica de cualquier celda i, [L]

$\eta \quad$ coeficiente de atenuación delaluz en el agua, $\left[L^{-1}\right]$

$\phi_{b r} \quad$ radiación emitida por la superficie del agua, [E]

$\phi_{e} \quad$ flujo de calor por evaporación, [E]

$\phi \quad$ flujo de calor por conducción, [E]

$\rho_{i, m}$ densidad del agua, $\left[M L^{-3}\right]$

$\theta \quad$ coeficiente de temperatura, [adim]

\section{Conclusiones}

Aunque hasta ahora existe un gran avance en la formulación de modelos para simular la dinámica de la calidad del agua en los embalses, el alto grado de complejidad de la estructura y dinámica de tales sistemas, aunado a su carácter estocástico natural, ha impedido la formulación de un modelo dinámico completo de aplicación general. En este sentido, la experiencia ha mostrado que es más conveniente la formulación de submodelos en términos de los factores básicos de los procesos limnológicos, estructurados de tal manera que conduzcan a un mejor entendimiento de tales procesos y permitan la cuantificación de los parámetros involucrados, con lo que se logra avanzar realmente en la estructura de modelos más completos. Así, partiendo de las ecuaciones de continuidad y suponiendo un flujo casi-permanente, se obtuvo un modelo numérico en el que prevalece el criterio de simplicidad para estudiar la evolución $O D$ y $D B O$ en los sentidos longitudinal yvertical de los embalses. El modelo es de fácil manejo y, en diferencia con los paquetes de cálculo disponibles en el mercado, se le pueden hacer los cambios necesarios para ajustarlo a las condiciones en que se realizan las mediciones en los casos reales; asimismo, sin mucha dificultad se le pueden incorporar las ecuaciones que describen la cinética de otros parámetros de calidad del agua.

\section{Referencias}

Chapra S.C y Reckhow K.H. (1983). Engineering Approaches for Lake Management, Vol. 2 Mechanistic modeling, Butterworth Publ., Boston.

De Victorica A.J. (1988). Modelo numérico para el análisis del régimen térmico en las presas, Ingeniería Hidráulica en México, Vol.III, Núm. 1, II época enero/abril, ISSN-0186-4076.

Hiriart G.L. y Pereyra D. (1980). Balance de energía agua-atmósfera y algunas aplicaciones. IX Congreso Latinoamericano de la IAHR, Venezuela, 1-9.

Kikkawa H. (1978). Reservoir Sedimentation. Proceedings of the First Semminary Japanese-American in Erosion and Sedimentation, chap. 8; Honolulu, Hawaii, USA.

Krenkel P.A. y Novotry V. (1980). Water Quality Management. Academic Press, N.Y. 
Metcalf y Eddy Inc. (1979). Wastewater Engineering: Treatment, Disposal, Reuse. Second Edition, McGraw-Hill Book Company, N.Y.

Orlob G.T. (1983). Mathematical Modeling of Water Quality: Streams, Lakes and Reservoirs. John Wiley \& Sons, Inc., N.Y.

Reid G.K. y Wood R.D. (1976). Ecology of Inland Waters and Estuaries. Second Edition, D. Van Nostrand Company, N.Y.

Ryan P. y Harleman D. (1973). An Analitycal and Experimental Study of Transient Cooling Pond Behaviour.
Massachusetts Institute of Technology, Report 161.

Tabor H. y Weinberger Z. (1981). Nonconvective Solar Pounds. In: Solar Energy Handbook, Kreider and Kreith, Eds., McGraw-Hill Book Company, N.Y.

Tennessee Valley Authority, TVA. (1972). Heat and Mass Transfer Between a Water Surface and the Atmosphere. Laboratory report No. 14, Water Resources Research.

Thomann R.V. (1972). Systems Analysis and Water Quality Management. McGraw-Hill Book Company, N.Y.

\section{Semblanza del autor}

Jorge L. de Victorica-Almeida. Obtuvo los grados de doctor y maestro en ingeniería ambiental en la División de Estudios de Posgrado de la Facultad de Ingeniería de la UNAM, y el de ingeniero químico en la Facultad de Química, UNAM. Inició sus actividades profesionales como investigador en el Instituto Mexicano del Petróleo y posteriormente en el Centro de Investigación y Entrenamiento para el Control de la Calidad del Agua de la entonces Secretaría de Recursos Hidráulicos. Desde 1979 labora como investigador en el Instituto de Ingeniería de la UNAM. Laboró como profesor en la UAM-Azcapotzalco, así como en la Facultad de Química de la UNAM y fungió como coordinador de la asignatura Manejo de Recursos Naturales en la Facultad de Biología de la Universidad Michoacana de San Nicolás de Hidalgo. Actualmente es profesor del Posgrado de la Facultad de Ingeniería y miembro del Comité Técnico de Evaluación de Proyectos de Investigación del CONACyT. Asimismo, es asesor técnico de la planta de tratamiento de aguas residuales de la UNAM. Sus líneas de investigación son la ingeniería de procesos para el tratamiento de aguas y aguas residuales, así como modelos matemáticos de calidad del agua en ríos, lagos y embalses. 